\title{
EARTH SCIENCE
}

\section{Coastal catastrophe in Phoenicia}

\begin{abstract}
The coast of Lebanon - Phoenicia of the ancient world - has seen a lot of action over the centuries, and not just from trading or warring peoples. This is a tectonically complex and dynamic region, and on 9 July, AD 551, a catastrophic earthquake and subsequent tsunami hit cities along some $180 \mathrm{~km}$ of coast running from Tripoli in the north to Tyre in the south. Contemporary accounts and archaeological evidence attest to the extent of the devastation.

Ata Elias and colleagues, based at IPG-Paris and Ifremer, have been exploring the geological context of the AD 551 earthquake and tsunami, and that of similar events in the same area. As they describe in Geology (A. Elias et al. Geology 35, 755-758; 2007), they have come up with a fresh line of evidence for the seismic particulars involved. The cause, they conclude, was
\end{abstract}

a sudden rupture of the 'Mount Lebanon thrust', a previously unidentified offshore fault about 150 $\mathrm{km}$ in length, which runs close to the Lebanese coast. This satellite image shows the region concerned.

The new data come from a geophysical survey involving a towed acoustic system that allowed the deep-sea floor to be imaged. The resulting pictures reveal dramatic submarine scarps and ruptures on the sea floor, akin to those resulting from large-scale seismic activity on land, that have broken through the thick blanket of sediment.

The continental shelf is unusually narrow in this region, and the fault comes as close as $8 \mathrm{~km}$ to the coastline. Over geological time, movement on this fault has been responsible for elevation of the Mount Lebanon range.

Evidence for recurrent seismic

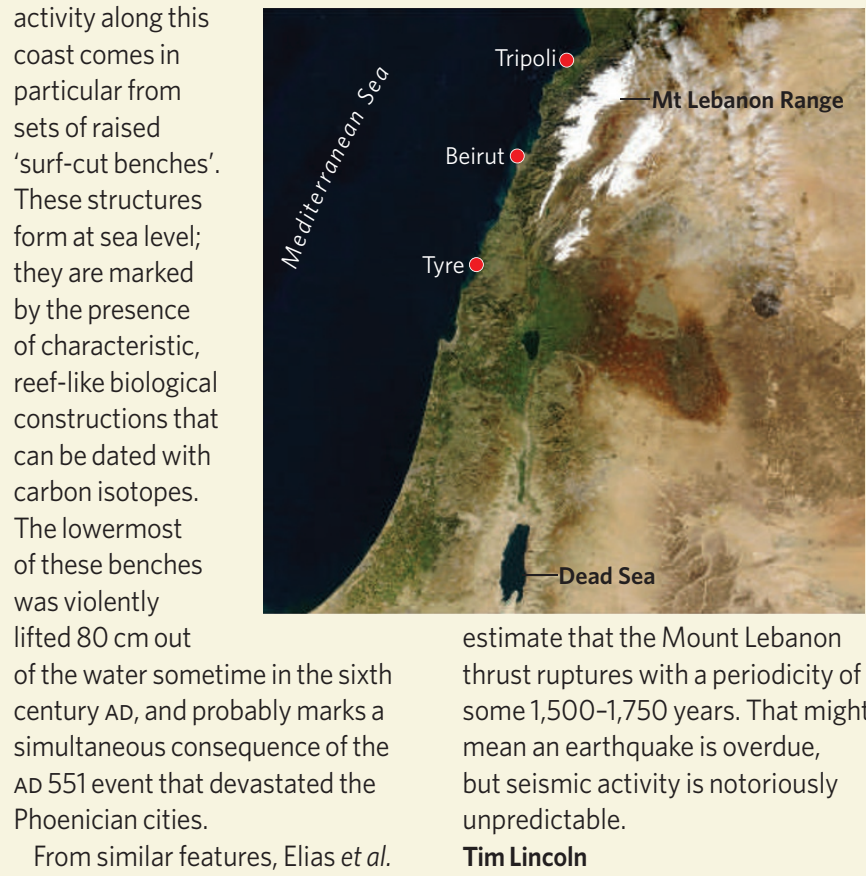

activity along this coast comes in particular from sets of raised 'surf-cut benches'. These structures form at sea level; they are marked by the presence of characteristic reef-like biological constructions that can be dated with carbon isotopes. The lowermost of these benches was violently lifted $80 \mathrm{~cm}$ out of the water sometime in the sixth century $A D$, and probably marks Phoenician cities. From similar features, Elias et al.

estimate that the Mount Lebanon eriodicity of $1,500-1,750$ years. That might Tim Lincoln variable. Hermann and colleagues therefore screened possible substrates for Tm0936 by modelling high-energy intermediates that would be formed during the enzyme reaction. These intermediates closely mimic the transition state for each substrate (Fig. 1a), and so provide better structures than those of the ground-state substrates for modelling binding interactions with the enzyme.

To limit the choice of possible substrates, Hermann et al. focused on naturally occurring compounds that are listed in the metabolite database of the Kyoto Encyclopedia of Genes and Genomes ${ }^{4}$. Knowing which chemical groups are recognized by AHS enzymes, they examined all of these metabolites and came up with a list of about 4,000 potential substrates. They then modelled tetrahedral intermediates of these compounds, including all the possible stereoisomers that arise in chiral molecules. Using computational methods, the authors docked all of the putative intermediates into a model of the active site of Tm0936 and identified the bestfitting candidates. They then used biochemical assays to test these candidates for catalytic activity and specificity, and identified several compounds that are excellent substrates for Tm0936. With the function of Tm0936 thus revealed, the authors were also able to assign the roles of 78 previously unannotated ORFs of AHS members found in many species. Furthermore, the reaction that Tm0936 catalyses could form part of a previously undiscovered degradation pathway.

Enzyme superfamilies are constantly being discovered $^{3}$. Using Hermann and colleagues' approach $^{2}$, it should be possible to determine the functions of members of these families that currently have unknown roles. For example, enzymes of the enolase superfamily (ENS) have a $(\beta / \alpha)_{8}$-barrel structure similar to that of the AHS, and their mechanism has been extensively studied ${ }^{3}$. They remove a hydrogen ion $\left(\mathrm{H}^{+}\right)$from carbon atoms adjacent to carboxylate groups $\left(\mathrm{CO}_{2}^{-}\right)$in substrates, generating an intermediate known as an enolate that is stabilized by metal atoms in the enzyme active site (Fig. 1b). This process changes the geometry of the reactive carbon atom from a tetrahedral arrangement of four groups about the carbon in the ground state to a planar arrangement of three groups about that atom in the high-energy intermediate. The planar geometry of the intermediate could be used as the basis of a metabolite search-pool. Chemical expertise is essential for choosing the best bond distances and charge distributions in the computer-generated intermediates, ready for the enzyme-docking analysis.

The AHS and ENS account for a large number of enzymes, so although Hermann and colleagues' method is labour intensive, it may be very useful in assigning enzyme function. Nevertheless, a few problems remain, as the authors have noted ${ }^{2}$. Proteins in the ENS are composed of two domains, one of which does not share amino-acid sequences with other superfamily members, even though it contributes to substrate specificity. Furthermore, multidomain structures often exist in different structural states: open (in the absence of substrate) and closed (during catalysis; that is, in the presence of a high-energy intermediate). Thus, available crystal structures may not be indicative of the closed state, which would be most relevant to the enzyme's reaction.

Another issue is our limited knowledge of metabolites; more of these must be identified if the authors' approach is to be effective. Finally, Hermann and colleagues' process must be developed into a higher-throughput method if it is to make an impact on this challenging area. Further experiments are required to see if their technique is truly general for all enzymes, but the indications are that it will provide an excellent complement to other methods for assigning roles to unknown ORFs.

JoAnne Stubbe is in the Department of Chemistry, Massachusetts Institute of Technology, Cambridge, Massachusetts 02139, USA. e-mail: stubbe@mit.edu

1. www.genomesonline.org

2. Hermann, J. C. et al. Nature 448, 775-779 (2007).

3. Gerlt, J. A. \& Babbit, P. C. Annu. Rev. Biochem. 70, 209-246 (2001).

4. Kanehisa, M. \& Goto, S. Nucleic Acids Res. 28, 27-30 (2000).

\section{Correction}

In the News \& Views article "Chemical biology: lons illuminated" by Christopher J. Chang (Nature 448, $654-655 ; 2007)$ an error crept into part $\mathbf{a}$ of the accompanying figure. The correct structure of $\mathrm{CaGF}$ can be found at http://pubchem.ncbi.nlm.nih.gov/ summary/summary.cgi?sid=24711513 